\title{
Multiarmed Spirals in Excitable Media
}

\author{
Bakthier Vasiev, ${ }^{1}$ Florian Siegert, ${ }^{2}$ and Cornelis Weijer ${ }^{1, *}$ \\ ${ }^{1}$ Department of Anatomy \& Physiology, University of Dundee, Dundee DD1 4HN, United Kingdom \\ ${ }^{2}$ Zoological Institute, University of Munich, Luisenstrasse 14, 80333 Munich, Germany
}

(Received 17 June 1996)

\begin{abstract}
Numerical studies of the properties of multiarmed spirals show that they can form spontaneously in low excitability media. The maximum number of arms in a multiarmed spiral is proportional to the ratio of the single spiral period to the refractoriness of the medium. Multiarmed spirals are formed due to attraction of single spirals if these spirals rotate in the same direction and their tips are less than one wavelength apart, i.e., a spiral broken not far from its tip can evolve into a 2-armed spiral. We propose this mechanism to be responsible for the formation of multiarmed spirals in mounds of the slime mold Dictyostelium discoideum. [S0031-9007(97)02750-6]
\end{abstract}

PACS numbers: 87.22. $-\mathrm{q}, 47.54 .+\mathrm{r}$

Target and spiral wave patterns are known to occur in excitable media. While target patterns result from periodic artificial stimulation (or alternatively occur in oscillatory media), spiral waves when initiated can indefinitely persist in excitable media. Examples of spiral waves are observed in a variety of physical, chemical, and biological systems [1]. The properties of single spiral waves as well as the conditions responsible for their formation have been studied in detail [2]. However, the problem of spiral interactions, especially the formation of multiarmed spirals, is less well investigated. Multiarmed spirals can be identified by the fact that all the arms of a multiarmed spiral propagate through a common territory and therefore form one high frequency wave source.

There are two important open questions associated with multiarmed spirals: How are they formed and are they stable? Multiarmed spirals never occur spontaneously in excitable chemical media such as the BelovsovZhabestinskii reaction; however, they can be constructed experimentally [3]. On the other hand, in a well investigated biological excitable system, the development of the cellular slime mold Dictyostelium discoideum (Dd), they can occur spontaneously [4]. Aggregation of starving cells is controlled by propagating spiral waves of the chemoattractant cAMP, which, upon mound formation, transform into multiarmed spirals (Fig. 1). In this Letter we investigate the conditions under which multiarmed spirals can spontaneously form, and suggest a mechanism for their formation. As to the stability of multiarmed spirals, it was suggested in [5] that multiarmed spirals are never stable since the arms repel each other. However, here we define the conditions in which the arms of a multiarmed spiral can rotate stably around one common core.

For calculations we have used FitzHugh-Nagumo equations [6],

$$
\begin{aligned}
& \partial g / \partial t=D \Delta g-k_{g} g(g-a)(g-1)-k_{r} r, \\
& \partial r / \partial t=(g-r) / \tau .
\end{aligned}
$$

These equations are commonly used to describe a prototype excitable medium. In our case, $g$ is taken to represent the concentration of the extra-cellular cAMP and $r$ the number of desensitized cAMP receptors in a field of aggregating Dd amoebae [7].

Calculations were performed in a two-dimensional $400 \times 400$ array using the explicit Euler method with a space step $h_{x}=0.5$ and time step $h_{t}=0.05$. Von Neumann's "no flux" boundary conditions were imposed on the boundaries of the medium. In the computations we have used the following set of parameters: $a=0.05$, $D=1, k_{g}=4.86, k_{r}=1.5$, and $\tau=5$. To test the numerical stability we performed computations with a twofold smaller value of the space step and fourfold smaller time steps. These showed less than $2 \%$ change in the velocity of single planar waves and less than $3 \%$ change in the period of spiral rotation. Therefore we conclude that the computations are stable for all practical purposes.

Figure 2 shows the influence of the density of randomly initiated spirals on the evolution of these spirals in a medium of given excitability and refractoriness. If the number of spirals initiated in the medium is small, i.e., the distances between their tips is big enough, the spirals interact only slightly. This type of interaction results in a drift of the spirals with respect to each other [Fig. 2(A)].
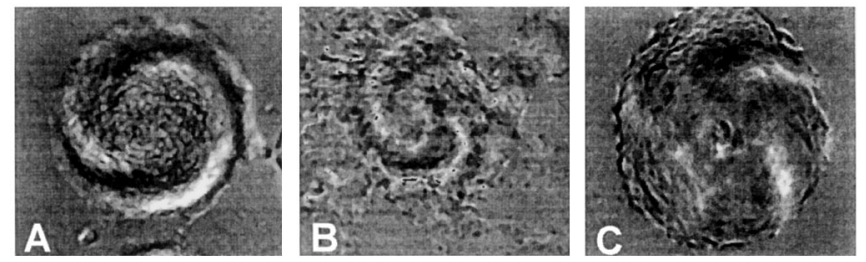

FIG. 1. (A) Two-armed, (B) three-armed, and (C) five-armed spirals of propagating optical density waves observed in mounds of Dictyostelium discoideum under dark field optics. The waves are due to light scattering changes caused by changes in the rate of chemotactic movement of individual cells in response to propagating spiral waves of cAMP. 


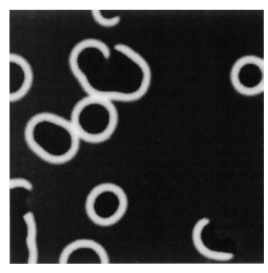

A $\quad t=25$

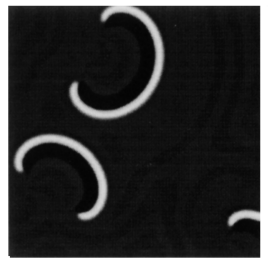

$t=250$

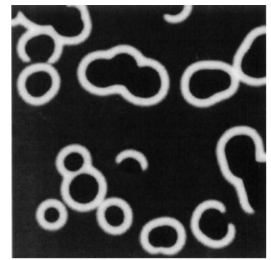

B $\quad t=25$

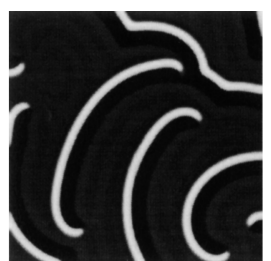

$\dagger=250$

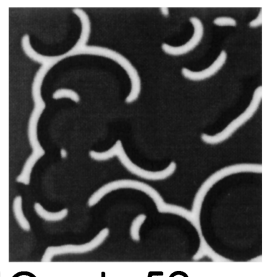

C $\quad t=50$

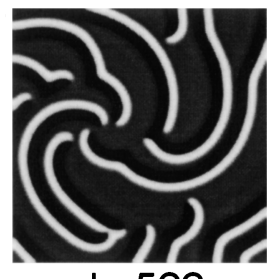

$t=500$

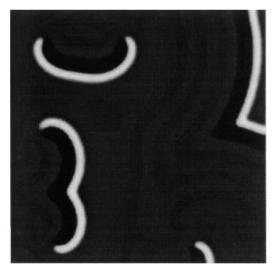

$t=1000$

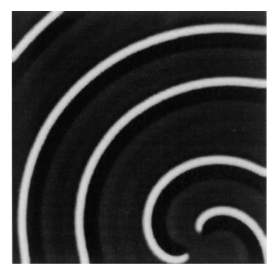

$t=1000$

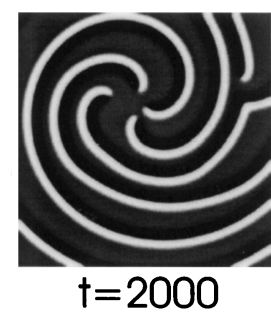

FIG. 2. Randomly distributed broken waves can evolve into single, 2-armed, or even multiarmed spirals. The number of arms in the multiarmed spirals depends on the initial density of the wave breaks. Broken waves were initiated by the addition of random noises $R(g+0.1)$ and $R 1(r+0.1)$ to the righthand sides of (1) and (2), respectively [8]. $R$ and $R 1$ are random numbers which vary in the range from -0.25 to 0.25 . Both values are changing in time (time step is 0.25 or $5 \mathrm{ht}$ ) and in space (space step is 2.5 or $5 h x$ ). This random noise was imposed during the initial part of the computation [to $t=11$ in (A), $t=12.5$ in (B), and $t=20$ in (C)]. Because of this noise, waves were formed and broken. The longer the noise is imposed, the more broken waves occur in the medium. The gray scale from black to white corresponds to the values of $g$ from -0.1 to 0.9 . In addition, black corresponds to values of $g$ lower than -0.1 and white to values higher than 0.9 (range of variation of $g$ during computations is from -0.27 to 0.92 ).

If the number of spirals initiated is higher, they interact more strongly. There are some cases where spirals rotate in the same direction and their tips are located not too far from each other. These spirals attract each other and form 2-armed spirals [Fig. 2(B)]. These 2-armed spirals emit excitation waves at twice the frequency of singlearmed spirals. As a consequence, they repel all single spirals until they are expelled from the medium.

If the number of spirals initiated in the medium is very high, even more than two spirals can attract each other. An example of this kind of strong interaction is shown in Fig. 2(C). First of all, almost all spirals rotating clockwise attract each other and form one multiarmed spiral. This multiarmed spiral quickly repels all the remaining single spirals from the medium. Later, the multiarmed spiral loses, one by one, most of its arms, repelling them from the medium as well. In the case shown in Fig. 2(C) only four arms remain.

The arms of the multiarmed spirals shown in Figs. 2(B) and $2(\mathrm{C})$ do not rotate around a fixed core, the tips all meander. However, it is possible to initiate multiarmed spirals whose arms rotate stably around a common core. In Fig. 3 we show 2-, 3-, and 5-armed spirals stably rotating in relatively high, intermediate, and low excitability media, respectively. The spiral core diameter increases with a decrease in the excitability of the medium. Decreasing the excitability results in an increase of the single spiral period as well as in the maximal number of arms of the multiarmed spiral $\left(N_{\max }\right)$ that can be initiated. The 5 -armed spiral initiated in low excitability medium is not stable in the higher excitable media shown in Figs. 3(A) and 3(B). The 2-armed spirals can rotate stably in any of the media shown.

In Fig. 4(a) a plot of maximal number of arms (with common core) versus the excitability of the medium is given. Excitability has been changed by the variation of parameter $k_{g}$ (rate of $g$ production). A decrease of $k_{g}$ in the given range results in a sharp increase in the rotation period of a single-armed spiral (almost $1000 \%$ ), a small decrease in the velocity of excitation waves (around 25\%), and has almost no effect on the refractoriness of the medium (10\%). Therefore, the maximum number of arms, $N_{\max }$, increases with an increase in the rotation period of a single spiral. The velocity of wave propagation for a multiarmed spiral is almost the same as for a single spiral.

We have found that an increase in the refractoriness of the medium (by variation of the relaxation time $\tau$ ) results in a decrease of $N_{\max }$. Figure 4(b) shows how a plane of period versus refractoriness is divided into regions

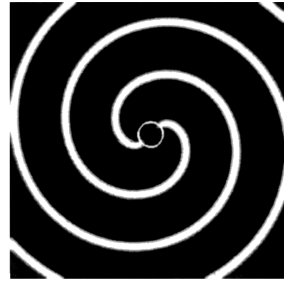

A

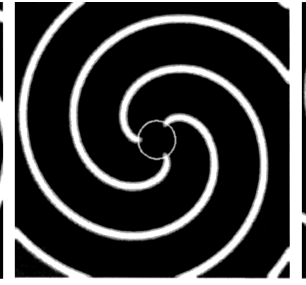

B

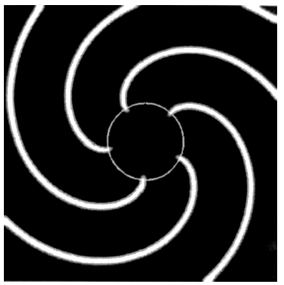

C
FIG. 3. Multiarmed spirals whose arms rotate stably around common cores are shown in media with different excitability. Excitability is changed by the variation of the production rate of $g: k_{g}=5.0$ in (A), $k_{g}=4.86$ in (B), and $k_{g}=4.7$ in (C). The possible number of arms increases with a decrease in the excitability of the medium. To obtain $n$-armed spirals we have initiated $n$ times a broken wave (piece of planar wave) with an interval $n$ times smaller than the period of a single spiral. The thin lines denote the trajectories of the spiral tips. To define the location of a spiral tip we have looked for a grid with $x y$ coordinates $(i, j)$ for which the four neighbors located at $(i, j \pm 1)$ and $(i \pm 1, j)$ satisfy the following four different conditions: $(g<0.4$ or $g>0.4)$ and $(r<0.4$ or $r>0.4)$. 

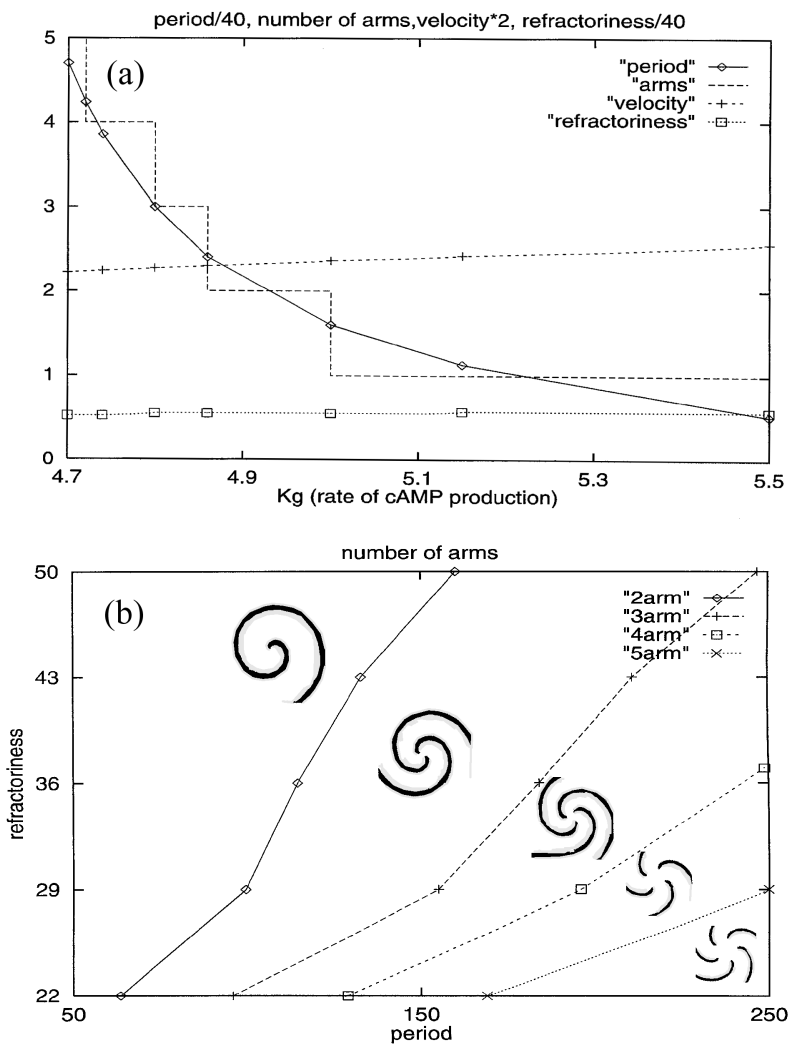

FIG. 4. (a) The maximal number of arms in a multiarmed spiral, period of a single-armed spiral, the velocity of the waves, and the refractoriness of the medium as functions of the rate of production of $g\left(k_{g}\right)$. The velocity was measured for single planar waves. Refractoriness was measured in a onedimensional medium as a minimal interval between waves in response to the lowest stimulus exciting the medium. $N_{\max }$ has been determined by checking the stability of $n$-armed spirals with an initial interval between arms equal to $T_{\mathrm{sp}} / n$. (b) Diagram showing the regions of occurrence of multiarmed spirals with $1,2,3,4$, and 5 arms as depending on the period of a single spiral and the refractoriness of the medium.

with different $N_{\max }$. The value of $N_{\max }$ can be estimated roughly from the relationship of the single-spiral period, $T_{\mathrm{sp}}$, to the refractoriness of the medium, Refr.

$$
N_{\max }=\operatorname{int}\left(A\left(T_{\mathrm{sp}}\right) T_{\mathrm{sp}} / \text { Refr }\right) .
$$

The factor $A\left(T_{\mathrm{sp}}\right)$ slowly increases from 1.5 to 1.8 for the increase in $T_{\mathrm{sp}}$ shown in Fig. 4(b). To obtain Fig. 4(b), we varied parameters $k_{g}$ and $\tau$. Changes in $N_{\max }$ due to the variation of the other model parameters, $a$ and $k_{r}$, are in good agreement with those shown in Fig. 4(b). An increase in parameter $a$ (resulting in an increase in $T_{\mathrm{sp}}$ and $N_{\max }$ as well as in a decrease in the velocity of waves and to negligible changes in the refractoriness) is very similar to a decrease in $k_{g}$. An increase in $k_{r}$ is qualitatively similar to a decrease in $\tau$, since both decrease velocity and refractoriness, and increase $T_{\mathrm{sp}}$ and $N_{\max }$.

The last point that we will address is the mechanism for the formation of multiarmed spirals in Fig. 2. They occur due to the attraction of single spirals rotating in the same direction, when the distance between them is small in comparison with the wavelength. This is illustrated in Figs. 5(A) and 5(B), which show the effects of the breaking of a spiral wave at different distances from the tip. Each broken spiral gives rise to three spirals. The spirals shown in Fig. 5(A') almost do not interact. There is a slow drift of spirals 2 and 3 which are very close to each other but rotate in opposite directions. There is no interaction between spirals 1 and 2 since they are far apart (initial distance is 90 space units). The spiral in Fig. 5(B) has been broken closer to its tip. As a result, spirals 1 and 2 (initial distance is 60 space units) attract each other and form a 2-armed spiral. The 2-armed spiral repels the third single-armed spiral rotating in the opposite direction, leading to the exclusion of spiral 3 from the medium. The attraction of spirals 1 and 2 is caused by the influence of
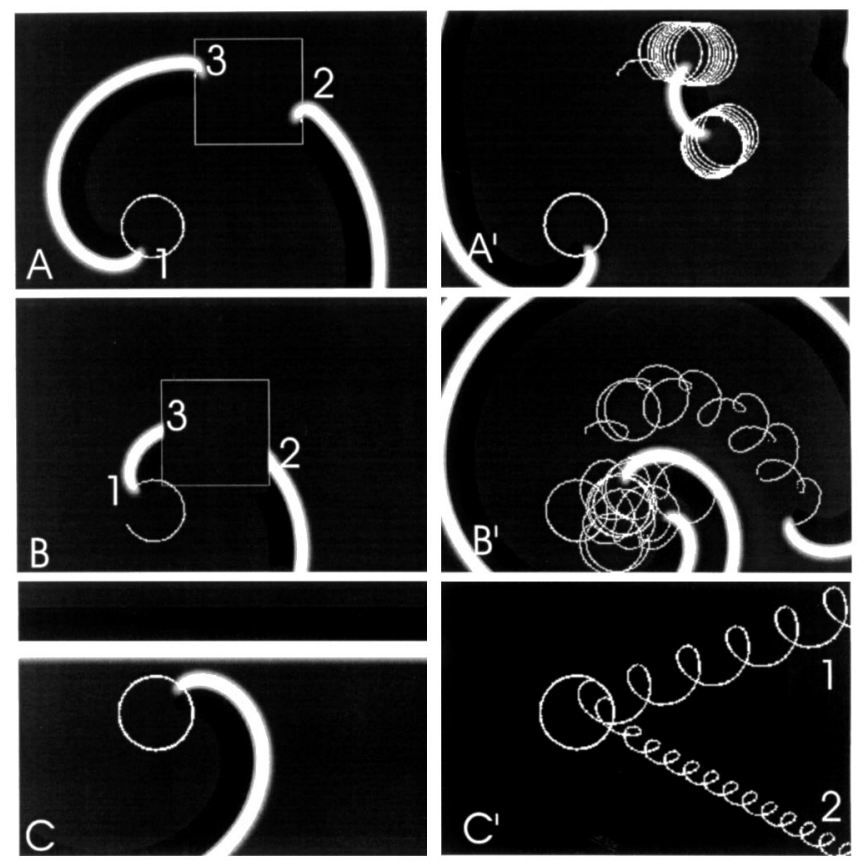

FIG. 5. (A), ( $\left.\mathrm{A}^{\prime}\right),(\mathrm{B}),\left(\mathrm{B}^{\prime}\right)$ A break of a single spiral can result either in the formation of new single spirals $(A)-\left(A^{\prime}\right)$ or to the formation of a 2-armed spiral (B)-(B'). A 2-armed spiral is formed when the break is located not too far from the spiral tip. In the medium shown the critical distance is 70-80 space units, while the wavelength of a single spiral is 110 space units. To introduce a break in the spiral, the values of both variables are reset to their rest values $(0)$ inside the rectangles marked in (A) and (B). $t=105$ in (A) and $t=80$ in (B) $(t=0$ is the time of initiation of the original spiral). The state of the media at $t=1000$ is shown in $\left(\mathrm{A}^{\prime}\right)$ and $\left(\mathrm{B}^{\prime}\right)$. The thin lines denote the tracks of spiral tips. (C), ( $\left.C^{\prime}\right)$ Interaction of a spiral with a sequence of planar waves propagating from the upper boundary of the medium. Because of the interaction, the spiral drifts. There is a vertical component of the drift which is in the direction of the planar wave source when the period of the planar waves is 65 time units [track 1 in $\left(C^{\prime}\right)$ ] and in the opposite direction when the period is 25 time units [track 2 in $\left(\mathrm{C}^{\prime}\right)$ ]. The period of the spiral is 96 time units. 
TABLE I. Periods of spirals 1 and 2 during the first nine loops of their rotation [Figs. 5(B) and $\left.5\left(\mathrm{~B}^{\prime}\right)\right]$.

\begin{tabular}{cccccccccr}
\hline \hline LOOP & 1 & 2 & 3 & 4 & 5 & 6 & 7 & 8 & 9 \\
\hline SPIRAL 1 & 64.5 & 82.3 & 74.2 & 62.3 & 87.5 & 98.5 & 87.7 & 96.2 & 104.3 \\
SPIRAL 2 & 98.6 & 60.5 & 81.1 & 95.1 & 96.5 & 93.7 & 95.5 & 96.2 & 96.4 \\
\hline \hline
\end{tabular}

the waves emitted by one spiral on the motion of the other spiral's tip. This influence is similar to that of waves emitted by a high frequency wave source on the spiral. It has been shown that the spiral will start to drift due to this influence [9]. In Figs. 5(C) and 5(C') the effect of a sequence of planar waves on a single spiral is shown. If the frequency of the planar waves is slightly higher than that of the spiral, the spiral shifts in the direction of a planar wave source, i.e., the spiral is attracted by this source [track 1 in Fig. 5(C')]. An increase in this frequency results in a shift from attraction to repulsion [track 2 in Fig. 5(C')].

We therefore expect that the spirals 1 and 2 in Fig. 5(B') attract each other since each spiral feels the other as a wave source of slightly higher frequency. That this is correct is shown in Table I which shows the periods of spirals 1 and 2 during the first nine loops of their rotation. Their periods have been detected by tracking of the motion of the spiral tips. The period of an undisturbed single spiral in this particular medium is 96.6 time units. It is seen that the interacting spirals rotate faster for the first four loops which corresponds to the time that they attract each other. Furthermore, it is seen that spiral 1 is, on average, faster than spiral 2 and therefore acts as a high frequency wave source. After this initial period of attraction the periods of both spirals are more stable and closer to the period of the single spiral (the last five loops).

The most important result reported in this Letter is that a multiarmed spiral can arise spontaneously from singlearmed spirals. We have found that they only form in low excitability media (in the model used, the period of single spiral should be at least 3 times higher than the refractoriness of the medium). Furthermore, the density of spirals initiated in the medium has to be high enough so that there are spirals rotating in one direction at distances less than the single-spiral wavelength. It is known that cAMP spiral waves can transform spontaneously into multiarmed spirals in the early stages of Dd mound formation (Fig. 1). However, it was not at all clear how they could form. Based on our calculations, we assume that at this stage the excitability of the cAMP relay system decreases, a change caused by a switch in the expression of high to low affinity cAMP receptors [10]. Furthermore, during this time, the mound is very inhomogeneous and irregularly shaped, and this could cause breaks of the original single-armed spiral. Such a broken spiral could then evolve into a multiarmed spiral in the same way as in Figs. 2(C) and 5(B).
In our computations we also observed multiarmed spirals whose arms rotate stably around a common core (Fig. 3). The multiarmed spirals observed so far did not seem to be stable [3]. Moreover, it was assumed in [5] that multiarmed spirals can never be stable. We now understand why the multiarmed spirals in [5] were unstable. The excitability of the computational media used in [5] was not low enough. The ratio of the singlespiral period to the refractoriness of the medium was 1.7 , which is not enough to get stable 2-armed spirals (see Fig. 4). Starting from random initial conditions, we found that the arms of the multiarmed spirals are meandering [Figs. 2(B), 2(C), and 5(B)]. We have shown, however, that it is possible to get arms rotating stably (for more than ten rotations) along the same core in the special case of completely symmetric initial conditions (Fig. 3).

We gratefully acknowledge the Alexander von Humboldt Foundation for a research fellowship to Bakthier Vasiev.

*Electronic address: cjweijer@anatphys.dundee.ac.uk

[1] Nonlinear Wave Processes in Excitable Media, edited by A. V. Holden, M. Marcus, and H. G. Othmer (Plenum Press, New York, 1991).

[2] V.S. Zukov, Modeling of Wave Processes in Excitable Media(Manchester University Press, Manchester, 1988).

[3] K. I. Agladze and V.I. Krinsky, Nature (London) 296, 424-426 (1982); O. Steinbock and S. C. Müller, Int. J. Bifurcation Chaos 3, 437-443 (1993).

[4] F. Siegert and C. J. Weijer, Curr. Biol. 5, 937-943 (1995); J. Rietdorf, F. Siegert, and C. J. Weijer, Dev. Biol. 177, 427-438 (1996).

[5] E. A. Ermakova, A. M. Pertsov, and E. E. Schnoll, Physica (Amsterdam) 40D, 185-195 (1989).

[6] R. FitzHugh, Biophys. J. 1, 445-466 (1961); I. Nagumo, S. Arimoto, and S. Yoshizava, Proc. IRE 50, 2061-2071 (1962).

[7] B. N. Vasiev, P. Hogeweg, and A. V. Panfilov, Phys. Rev. Lett. 73, 3173-3176 (1994).

[8] E. Palson and E. C. Cox, Proc. Natl. Acad. Sci. U.S.A. 93, 1151-1155 (1996).

[9] V.I. Krinsky and K. I. Agladze, Physica (Amsterdam) 8D, 50-56 (1983); Ye. A. Yermakova, V.I. Krinskii, A. V. Panfilov, and A.M. Pertsov, Biophizika 31, 318-323 (1986) [Biophys. 31, 348 (1986)].

[10] R. D. M. Soede, R. H. Insall, P. N. Devreotes, and P. Schaap, Development 120, 1997-2002 (1994). 